\title{
APLICAÇÃo dA DISTRIBUIÇÃO DE WEBER AO PESO DE PINHAS VERDES ${ }^{1}$
}

\author{
Saulo Henrique Weber ${ }^{2}$, Julio Eduardo Arce ${ }^{3}$ e Sylvio Péllico Netto ${ }^{4}$
}

\begin{abstract}
RESUMO - O objetivo deste trabalho foi verificar a eficiência da Distribuição de Weber quando aplicada aos dados de frequências de pesos de pinhas. Foram coletadas e pesadas 40 pinhas verdes, na Região Metropolitana de Curitiba. Os pesos foram distribuídos em seis classes com intervalo de $90 \mathrm{~g}$. A Distribuição de Weber ajustouse aos dados dos pesos das pinhas, de acordo com o teste de Kolmogorov-Smirnov. Os valores esperados pela distribuição, a média e a moda calculadas resultaram em valores próximos aos observados, mostrando que as expressões desenvolvidas foram eficientes. A facilidade para ajustar o modelo ora apresentado, o cálculo da média, a variância, o desvio-padrão e os pontos de inflexão, por meio das expressões desenvolvidas, são vantagens dessa distribuição. Devido à sua flexibilidade, pode-se afirmar que essa técnica pode ser aplicada a diversas distribuições de dados do setor florestal, bem como a outras séries de dados biológicos.
\end{abstract}

Palavras-chave: Funções de densidade de probabilidade, classes diamétricas, projeção e predição.

\section{APPLICATION OF THE WEBER DISTRIBUTION TO THE GREEN PINE CONES WEIGHT}

\begin{abstract}
The objective of the present work was to verify the efficiency of the Weber Distribution, when applied to the weight of pine cones. Forty green pine cones were collected and weighed in the Metropolitan Region of Curitiba. The weights were distributed into 6 classes, with 90 gram interval. It is concluded that the Weber Distribution adjusted to the data, in accordance with the Kolmogorov-Smirnov Test. The expected values for the adjusted distribution, average and mode had resulted in values close to the observed ones, showing that the developed expressions are efficient. The facility to adjust the proposed model, and the calculation of the average, variance, standard deviation and inflection points, by means of the developed expressions, are advantages of this distribution. Due to its flexibility, it is possible to affirm that this model can be applied to several forest distributions, as well as to other biological data.
\end{abstract}

Keywords: Probability density function, pine cone weight and asymmetric distributions.

\section{INTRODUÇÃO}

O gênero Araucaria $\mathrm{L}$. Jussieu tem sua origem há cerca de 200 milhões de anos e é composto por 19 espécies, que ocorrem no Hemisfério Sul (Austrália, Papúa-Nova Guiné, Nova Caledônia, Vanuatu, Ilha Norfolk, Brasil, Chile e Argentina).

A espécie Araucaria angustifolia é nativa no Brasil e possui ampla área de distribuição, contribuindo para que o pinheiro-do-paraná se diferencie em raças locais ou ecotipos (REITZ e KLEIN, 1966).
A araucária (Araucaria angustifolia (Bert.) $\mathrm{O}$. Ktze.) é uma espécie de grande importância cultural, social e econômica para o Estado do Paraná e predomina em um dos mais importantes biomas do sul do Brasil - a Floresta Ombrófila Mista (floresta com araucária) (ANJOS et al., 2004). Essa espécie pertence à ordem Coniferae, da classe Coniferopsida, família Araucariaceae e é comumente conhecida como pinheiro-do-paraná, pinheiro-brasileiro ou brazilian pine (CARVALHO, 1994; REITZ e KLEIN; REIS, 1988; LORENZI, 2000).

\footnotetext{
${ }^{1}$ Recebido em 07.11.2007 e aceito para publicação em 23.06.2009.

${ }^{2}$ Universidade Estadual do Centro-Oeste, Santa Cruz, 85015-430 - Guarapuava, PR - Brasil. E-mail: <sauloweber@ gmail.com>.

${ }_{3}^{3}$ Departamento de Ciências Florestais, Universidade Federal do Paraná. E-mail: <jarce @ floresta.ufpr.br>.

${ }^{4}$ Pontifícia Universidade Católica do Paraná, Centro de Ciências Agrárias e Ambientais, São José dos Pinhais, PR - Brasil. E-mail: <pellico.sylvio@pucpr.br>.
} 
A floresta com araucária encontra-se bastante reduzida e fragmentada, com remanescentes localizadas principalmente em propriedades rurais. Estudos recentes mostram que as áreas consideradas primárias nas décadas de 1970 - 80 não existem mais, restando somente florestas em estágio avançado, médio e inicial de sucessão (FUPEF, 2001). Dos 20 milhões de hectares originalmente cobertos pela floresta de araucária, restam, atualmente, cerca de $2 \%$ dessa área. Particularmente no Estado do Paraná, as serrarias e o uso industrial foram as principais fontes responsáveis pelo desmatamento (GURGEL FILHO, 1990).

Atualmente, a araucária é incluída como espécie vulnerável na lista oficial da flora nacional ameaçada de extinção, com manejo restrito pela legislação vigente e controlado pelos órgãos oficiais (CARVALHO, 1994; PARANÁ, 1995). A exploração de sua madeira está restrita, sendo permitido somente o corte das árvores com DAP igual ou superior a $40 \mathrm{~cm}$, mediante a aprovação de Plano de Manejo.

A araucária é perenifólia, com altura variando de 10 a 35 m e DAP entre 50 e $120 \mathrm{~cm}$, quando adulta (CARVALHO,1994; REITZet al., 1988). O tronco é reto e quase cilíndrico, ramificando-se em pseudoverticilos, com acículas simples, alternas, espiraladas, lineares a lanceoladas e coriáceas, podendo chegar a $6 \mathrm{~cm}$ de comprimento por $1 \mathrm{~cm}$ de largura. Possui casca grossa (até $10 \mathrm{~cm}$ de espessura), de cor marrom-arroxeada, persistente, áspera e rugosa (CARVALHO, 1994).

As flores são dioicas, sendo as femininas em estróbilo, conhecida popularmente como pinha, e as masculinas são cilíndricas, alongadas e com escamas coriáceas, tendo comprimento variando entre 10 e $22 \mathrm{~cm}$ e diâmetro entre 2 e 5 cm (MATTOS, 1994).

Os frutos da araucária são, botanicamente, classificados como pseudofrutos, reunidos em estróbilo feminino, ou pinha (ovário), com 10 a $25 \mathrm{~cm}$ de diâmetro, composto de 700-1.200 escamas, com número variável de semente (5 a 150) e com até $4.700 \mathrm{~g}$ de peso, que perdem a viabilidade gradualmenteem 120dias(CARVALHO, 1994). Popularmente, os frutos da araucária são conhecidos como pinha.

O pinheiro-do-paraná, quanto ao grupo sucessional, é uma espécie pioneira e heliófila, que se estende sobre os campos, formando novos capoeirões, mas sendo beneficiada por leve sombreamento na fase de germinação e crescimento até 2 anos (REITZ e KLEIN,1966).
Considerando os aspectos fitossociológicos, $A$. angustifolia apresenta regeneração fraca, tanto no interior da floresta quanto em ambientes pouco perturbados, e ocorre associada às espécies dos gêneros Ilex (erva-mate), Ocotea (imbuia) e Podocarpus (pinheirobravo).

Mesmo sendo uma espécie exclusiva da Floresta Ombrófila Mista, o pinheiro-do-paraná ocorre em áreas de tensão ecológica com a Floresta Estacional Semidecidual e Floresta Ombrófila Densa, bem como em refúgios na Serra do Mar e Serra da Mantiqueira. No decorrer dos períodos geológicos, A. angustifolia apresentou dispersão geográfica bastante diversa da atual, pois foram encontrados fósseis no Nordeste brasileiro (IBGE, 1992).

A araucária interage intensamente com a fauna, que constitui elemento muito importante para a dispersão das sementes. Entre esses animais, destacam-se os roedores e as aves. Alberts (1992) citou, entre os roedores, as cotias, as pacas, os ouriços, os camundongos e os esquilos. Entre as aves, são citados por SolórzanoFilho e Kraus (1999) o papagaio-de-peito-roxo e a gralhapicaça; em Minas Gerais, Bustamante (1948) citou os airus, a gralha- azul e os tucanos.

Trata-se de uma planta dioica (há árvores femininas e masculinas), podendo ser monoica quando submetida a traumas ou doenças. Há predominância de pinheiros masculinos, tanto em áreas de ocorrência natural quanto em plantios (BANDEL e GURGEL, 1967). A floração feminina ocorre o ano todo e a masculina, de agosto a janeiro.

A polinização é predominantemente anemocórica (pelo vento) e, dois anos após esse evento, as pinhas amadurecem (REITZ et al., 1998).

Em plantios, a produção de sementes (pinhões) se inicia entre 10 e 15 anos, enquanto nas populações naturais essa fase se inicia a partir do vigésimo ano. Iniciada a produção de sementes, a árvore produz em média 40 pinhas por ano ao longo de toda a sua vida (mais de 200 anos).

As sementes de araucária são recalcitrantes, ou seja, perdem a viabilidade ao serem desidratadas, dificultando sua conservação por longos períodos. Suiter Filho (1966), citado por Carvalho (1994), recomendou a conservação dessas sementes em ambientes com umidade relativa acima de $80 \%$, pois 
a queda do poder germinativo é retardada. Nessas condições, após 60, 90 e 120 dias de armazenamento as sementes apresentaram poder germinativo médio de $75 \%, 45 \%$ e $45 \%$, respectivamente.

Krügel (1992) afirmou que em cada pinha é possível encontrar de 60 a 80 pinhões, com cerca de 125 a 200 pinhões por $\mathrm{kg}$.

Segundo Schumacher et al. (2004), em experimento realizado em povoamento de Araucaria angustifolia com 17 anos de idade no Município de Pinhal Grande, RS, a deposição de serapilheira foi de $6,96 \mathrm{Mg} / \mathrm{ha}$, sendo $74 \%$ acículas e $26 \%$ galhos.

De acordo com Schaaf et al. (2006), em pesquisa realizada em nove parcelas de 1 ha cada, em uma Floresta Ombrófila Mista no Município de São João do Triunfo, PR, em 1979, 11,6\% dos indivíduos adultos de araucária encontravam-se nas classes diamétricas acima de 50 $\mathrm{cm}$, enquanto em 2000 esses totalizavam $25,42 \%$.

Uma distribuição de probabilidade ou de frequências relativas é a síntese do agrupamento de dados em um espaço amostral; mostra a proporção das vezes em que a variável aleatória tende a assumir cada um dos diversos valores (STEVENSON, 1981).

Função-densidade de probabilidade contínua, denotada por fdp, são limites de infinita sequência de distribuições discretas, cujas variáveis formam um conjunto enumerável de valores.

O objetivo principal deste trabalho foi verificar a eficiência da Distribuição de Weber, quando aplicada aos dados de frequências de pesos de pinhas, uma vez que esse estudo não se encontra ainda disponível na literatura. Os objetivos específicos foram: utilizar as equações desenvolvidas para o cálculo de média, variância, moda e pontos de inflexão; comparar seus resultados aos obtidos empiricamente; e estudar o comportamento da distribuição dos pesos das pinhas verdes. Conhecer o comportamento de sua distribuição representa avanço para a conservação da espécie, bem como estabelece parâmetros que viabilizarão a sustentabilidade de plantios comerciais para fins de coleta de sementes, formando-se pomares.

\section{MATERIAL E MÉTODOS}

Foram coletadas 40 pinhas verdes na Região Metropolitana de Curitiba, nos Municípios de São José dos Pinhais, Fazenda Rio Grande e Tijucas do Sul, cuja formação é Floresta Ombrófila Mista, com altitude média de $900 \mathrm{~m}$. Cada pinha foi pesada com balança semianalítica marca Metler-Toledo. O clima da região, de acordo com a classificação de Köeppen, é do tipo cfb (temperado húmido com verão temperado), com ocorrência de geadas. Neste trabalho, foi utilizada a distribuição de frequências dos pesos das pinhas, aos quais foi ajustado a Distribuição de Weber. A Distribuição de Weber foi originalmente desenvolvida para representar a distribuição de alturas da regeneração natural do sassafrás (Ocotea odorifera (Vell.) Rower) existente na Fazenda Experimental Gralha Azul, localizada no Município de Fazenda Rio Grande (WEBER, 2006). No desenvolvimento daquela dissertação foi salientada a flexibilidade do modelo, e, dessa forma, este trabalho utilizou uma distribuição de dados diferente, a fim de apresentar outra aplicação.

O número de classes foi determinado, empiricamente, utilizando-se a regra de Sturges (equação 01 ), em que $K$ é o número de classes e $n$, a quantidade de dados (VIEIRA, 1991).

$$
K=1+3,322 \cdot \log (n)
$$

O valor calculado pela equação 7, com $n$ igual a 40 (número de pinhas utilizadas), foi de 6,32. Assim, conforme orientações descritas por Vieira (1991) foi utilizado o próximo valor inteiro como número de classes, ou seja, 7. O maior valor observado foi de 847,20 g e o menor, de 249 g; entretanto, foi tomado como menor limite inferior o valor de $240 \mathrm{~g}$, com o objetivo de facilitar o processo de distribuição das pinhas em classes de pesos. Dessa forma, a amplitude observada foi de 607,20. O resultado da divisão desse valor por 7 (número de classes determinado com base na fórmula de Sturges) é 86,74. O intervalo de classe utilizado foi de $90 \mathrm{~g}$, a fim de facilitar os cálculo.

A Distribuição de Weber foi ajustada por meio de regressão não linear, segundo o procedimento de Marquadt, pelo método de mínimos quadrados, com múltiplas iterações, de acordo com Marquadt (1963), utilizando-se o software Table Curve $2 d$.

A maioria dos algoritmos utilizados para estimar coeficientes não lineares pelo método de mínimos quadrados centra-se sobre uma de duas aproximações. No entanto, o modelo pode ser expandido como uma série de Taylor e as correções dos parâmetros, calculadas a cada iteração na suposição de

R. Árvore, Viçosa-MG, v.33, n.5, p.865-872, 2009 
linearidades locais. Entretanto, podem ser utilizadas modificações do método gradiente. O método de Marquadt utiliza o método de máxima verossimilhança, que executa uma ótima interpolação entre o método da série de Taylor e o método gradiente. A interpolação é baseada em máxima verossimilhança, na qual a série de Taylor truncada fornece uma representação adequada de um modelo nãolinear (MARQUADT, 1963).

\section{Seja}

$E(y)=f\left(x_{1}, x_{2}, \cdots, x_{m} ; \beta_{1}, \beta_{2}, \cdots \beta_{k}\right)=f(\mathrm{x}, \beta)$ o modelo a ser ajustado aos dados, em que $x_{1}, x_{2}, \cdots, x_{m}$ são as variáveis independentes, $\beta_{1}, \beta_{2}, \cdots \beta_{k}$ são os $k$ parâmetros e $E(y)$, o valor esperado da variável $y$. Denotando-se os pontos amostrados como $\left(Y_{i}, X_{1 i}, X_{2 i}, \cdots, X_{n i}\right), \quad i=1,2, \cdots, n$, o problema consiste em computar essas estimativas dos parâmetros que minimizem $\phi=\sum_{i=1}^{n}\left[Y_{i}-\hat{Y}_{i}\right]=\|Y-\hat{Y}\|^{2}$, em que $\hat{Y}_{i}$ é o valor de $y$ predito por $E(y)$ no $i$-ésimo ponto amostrado (MARQUADT, 1963).

O ajuste do modelo foi avaliado segundo o teste de Kolmogorv-Smirnov, que consiste em comparar a máxima diferença entre a frequência observada e a frequência estimada dividida pelo número de observações. Pode ser obtido pela equação 2:

$$
d_{\text {calc }}=\frac{\left(\max \left|F_{o}(X)-F_{e}(X)\right|\right)}{n}
$$

Em que $F_{o}(X)$ é a frequência observada acumulada, $F_{e}(X)$ a frequência esperada acumulada - estimada peló modelo - e $n$ o número de observações.

Se $d_{\text {calc }}$ for menor que o valor limite tabelado, aceita-se o ajuste. Se $n$ for menor que 50, para $\alpha=5 \%$, determina-se o valor crítico por meio da equação 3 .

$\alpha=5 \% \rightarrow d_{\text {crit }}=1,36 \cdot \sqrt{\frac{1}{n}}$

\section{DISTRIBUIÇÃO DE WEBER} A Distribuição de Weber é uma função cujo
domínio é o conjunto dos números reais positivos $\left(R^{+}\right)$, ou seja, $\quad 0 \leq x<\infty, \quad d \geq a+1, \quad d \neq a+2$, $d \neq a+3, a>0, b>0$ e $c>0$, definida pela equação 4 (WEBER, 2006). O comportamento da distribuição pode ser visualizado na Figura 1, de acordo com a variação de seus coeficientes.

$$
f(x)=\frac{x^{a}}{(b+c \cdot x)^{d}}
$$

A média aritmética e variância podem ser calculadas pelas expressões 5 e 6 :

Média aritmética $=\frac{b(a+1)}{c(d-2-a)}$ eq. 05

Variância $=-\frac{(a+1) b^{2}(d-1)}{c^{2}(a+3-d)(a+2-d)^{2}}$ eq. 06

O desvio-padrão é obtido extraindo-se a raiz quadrada da variância (eq. 7):

Desvio padrão $=\frac{b}{c(a+2-d)} \sqrt{-\frac{(a+1)(d-1)}{(a+3-d)}}$

eq. 07

A moda e os pontos de inflexão, quando existirem, poderão ser calculados utilizando-se as fórmulas das equações 8 e 9:

Moda $=\frac{a b}{c(d-a)}$ eq. 08 Pontos de inflexão $=\frac{b}{c} \cdot \frac{a^{2}-a d-a \pm \sqrt{-a d(a-d-1)}}{\left(a-d-(a-d)^{2}\right)}$ eq. 09 


\section{RESULTADOS E DISCUSSÃO}

Os dados observados dos pesos das pinhas foram agrupados em uma distribuição de frequências, conforme apresentados na Tabela 1 e representados no gráfico 1.

A Distribuição de Weber ajustada aos dados do peso dos pinhões está apresentada na equação 10 . Sua representação, bem como dos pontos observados, estão no gráfico 02 . Salienta-se que a probabilidade em uma distribuição acumula-se no centro de classe.

$f(x)=\frac{x^{10,483175}}{(2,473611+0,001349 \cdot x)^{54,877727}}$

O valor calculado do teste de Kolmogorov-Smirnov foi de 0,10 , enquanto o valor tabelado para $\alpha=5 \%$ foi de 0,21 ; logo, a distribuição foi considerada aderente aos dados observados. As frequências observadas e esperadas pelo modelo estão apresentadas na Tabela 2 .

Na Tabela 3 estão apresentados a média, a variância, o desvio-padrão e a moda observados e calculados pelas expressões desenvolvidas para a Distribuição de Weber.

Os pontos de inflexão do modelo de Weber aplicada à distribuição de frequências de pesos de pinhas, utilizando-se a expressão desenvolvida, foram de 285,98 e 580,08 g. É possível observar, nas Tabelas 2 e 3, que a distribuição ajustada resultou em valores esperados, média e moda próximos aos valores observados.

O uso de distribuições probabilísticas permite calcular a probabilidade de ocorrência de pinhas em qualquer intervalo de peso. Isso permite fazer prognoses mais precisas sobre a quantidade de pinhões produzidos por um indivíduo adulto, objetivando-se a coleta para fins conservacionistas e econômicos, uma vez que se espera que exista relação entre o peso das pinhas e o número de pinhões viáveis nelas contidas.

Tabela 1-Distribuição de frequências de pesos das pinhas. Table 1 - Frequency distribution of the pine cone weights. Pesos (g) Freqüência absoluta Freqüência acumulada

\begin{tabular}{lcc}
\hline $240-329$ & 5 & 5 \\
$330-419$ & 10 & 15 \\
$420-509$ & 8 & 23 \\
$510-599$ & 9 & 32 \\
$600-689$ & 6 & 38 \\
$690-779$ & 1 & 39 \\
$780-870$ & 1 & 40 \\
\hline
\end{tabular}

Tabela 2-Valores observados e esperados pela distribuição. Table 2 - Observed and expected values by the distribution.

\begin{tabular}{ccc}
\hline Peso $(\mathrm{g})$ & Freqüência observada & Freqüência esperada \\
\hline 285 & 5 & 4,75 \\
375 & 10 & 8,45 \\
465 & 8 & 9,05 \\
555 & 9 & 7,10 \\
645 & 6 & 4,55 \\
735 & 1 & 2,54 \\
825 & 1 & 1,30 \\
\hline
\end{tabular}

Tabela 3 - Média, variância, desvio-padrão e moda.

Table 3 - Average, variance, standard deviation and mode.

\begin{tabular}{lcc}
\hline & Observado & $\begin{array}{c}\text { Calculado pela } \\
\text { distribuição }\end{array}$ \\
\hline Média $(\mathrm{g})$ & 483,0000 & 496,7171 \\
Variância $\left(\mathrm{g}^{2}\right)$ & 17944,6154 & 27965,4814 \\
Desvio padrão $(\mathrm{g})$ & 133,9575 & 167,2288 \\
Moda $(\mathrm{g})$ & 352,5000 & 433,0323 \\
\hline
\end{tabular}

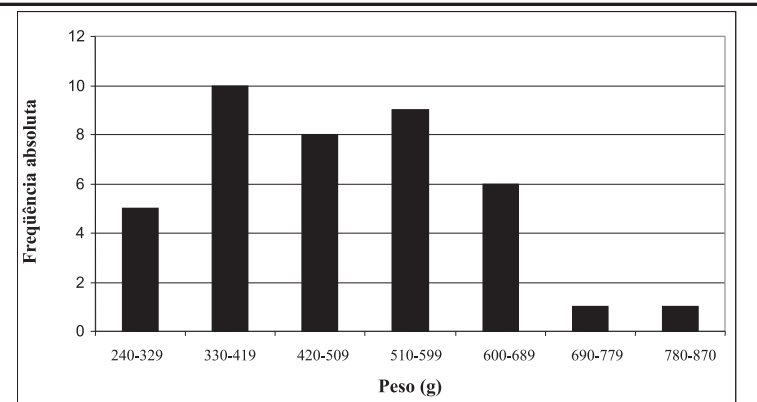

Figura 2 - Representação da distribuição de frequências dos pesos das pinhas.

Figure 2 - Representation of the frequency distribution of the pine cone weights.

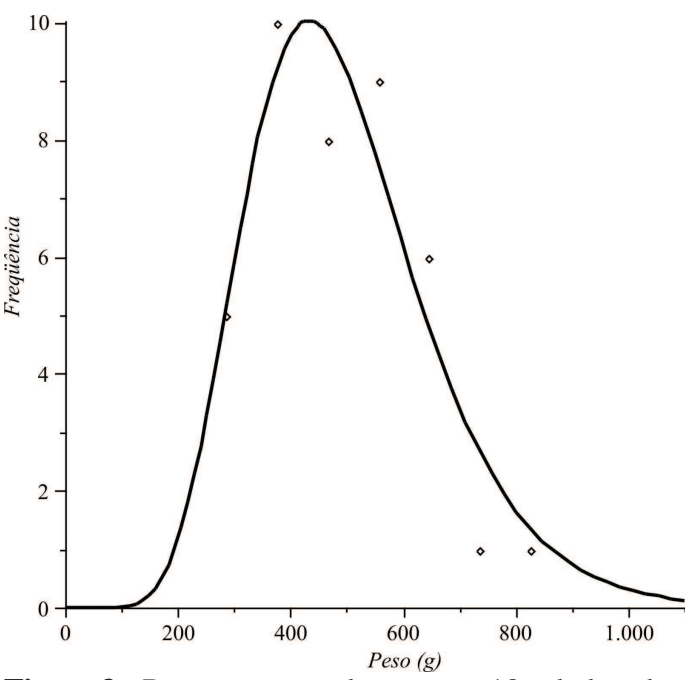

Figura 3 - Representação da equação 10 e dados observados dos pesos das pinhas.

Figure 3-Representation of equation 10 and observed data of the pine cone weights.

R. Árvore, Viçosa-MG, v.33, n.5, p.865-872, 2009 


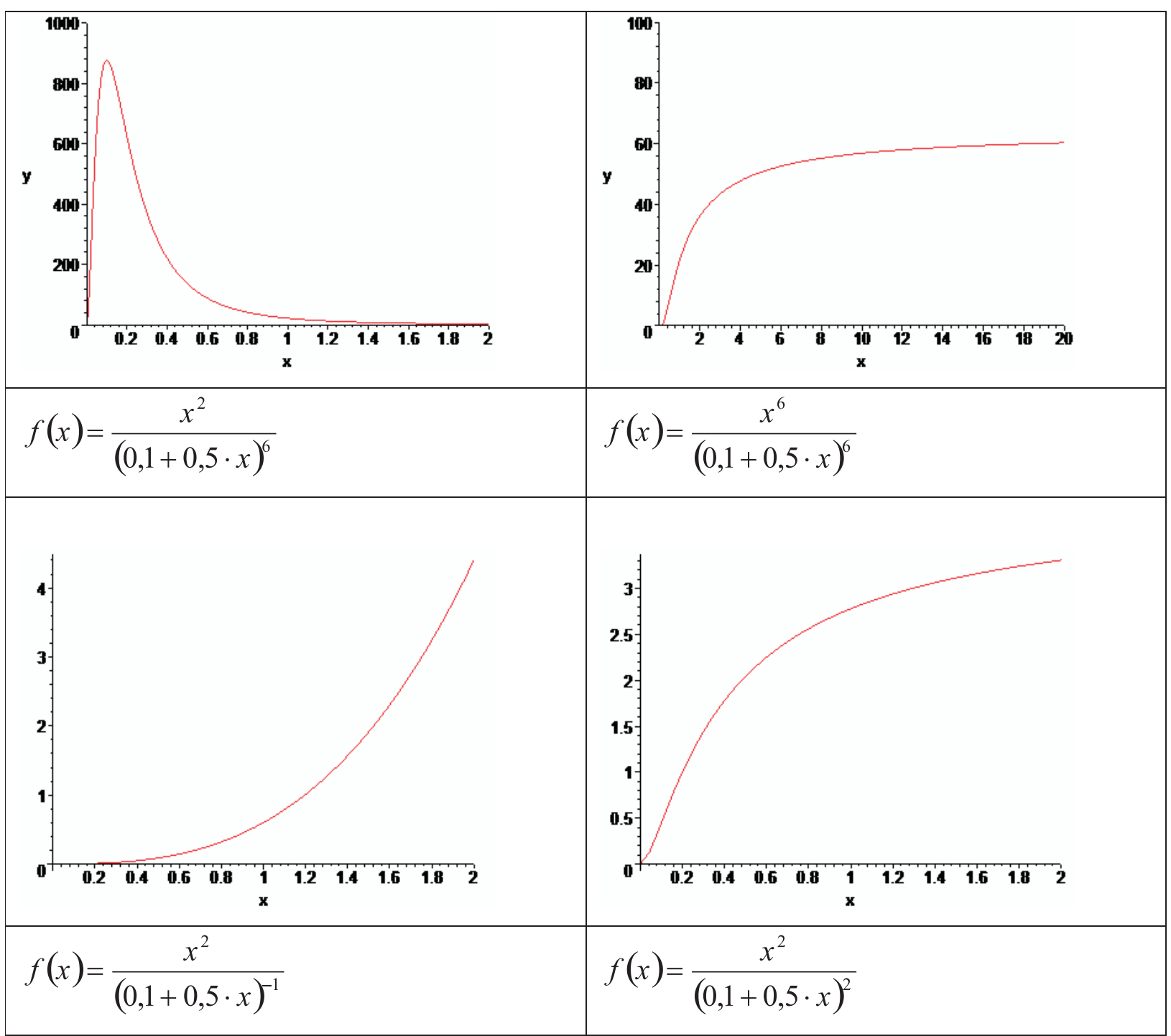

Figura 1 - Forma da Distribuição de Weber.

Figure 1 - Shape of Weber Distribution.

\section{CONCLUSÕES}

De acordo com os resultados deste estudo, é possível concluir que a Distribuição de Weber se ajustou satisfatoriamente aos dados dos pesos das pinhas, de acordo com o teste de Kolmogorov-Smirnov. Observou-se que a média e desvio-padrão calculados por meio das equações desenvolvidas para a Distribuição de Weber (496,71 g e 167,23 g, respectivamente) resultaram em valores próximos aos estimados com base na amostra (483,00 g e 133,96 g, respectivamente), com diferença de $13,71 \mathrm{~g}$ e $33,27 \mathrm{~g}$, respectivamente, nas estimativas desses dois parâmetros. Com relação à moda estimada pela distribuição e com base na amostra (433,03 g e $352,50 \mathrm{~g}$, respectivamente), foi observada uma diferença relativamente grande $(80,53 \mathrm{~g})$, entretanto, se pode concluir que o ajuste foi bom, uma vez que esse

R. Árvore, Viçosa-MG, v.33, n.5, p.865-872, 2009 
representou uma situação média, não sendo tendencioso a representar a moda e gerar resultados não satisfatórios para os demais pontos observados. A Distribuição de Weber apresentou dois pontos de inflexão, um em 285,98 g e outro em 580,08 g, o que representa duas mudanças de concavidade.

O modelo ora apresentado tem como vantagens: a) ser facilmente ajustada pelo software Table curve; e b) ter desenvolvidas as expressões para o cálculo da média, variância, desvio-padrão e pontos de inflexão.

Devido à flexibilidade da Distribuição de Weber, pode-se afirmar que essa pode ser aplicada a diversas distribuições de dados do setor florestal, bem como de outras séries de dados biológicos.

Neste trabalho, apresentou-se um primeiro estudo sobre a distribuição de pesos de pinhas verdes, entretanto é necessário que sejam feitas avaliações em outras condições edafoclimáticas. Estudos futuros devem considerar a alta variação de frequência do peso das pinhas.

\section{REFERÊNCIAS}

ALBERTS, C. C. O esquilo e o Pinheiro-do-Paraná: uma interação. Revista do Instituto Florestal, v.4, pt.4, p.1215-1216, 1992. (Edição dos Anais do Congresso Florestal de Essências Nativas, 2., 1992, São Paulo)

ANJOS, A. Análise do padrão de distribuição espacial da araucária (Araucaria angustifolia) em algumas áreas no Estado do Paraná, utilizando a função K de Ripley. Scientia Forestalis. n.66, p.38-45, 2004.

BANDEL, G.; GURGEL, J. A. A. Proporção do sexo em Araucaria angustifolia (Bert.) O. Ktze. Silvicultura em São Paulo, v.6, n. único, p.209-220, 1967.

BUSTAMANTE, I. L. F. Notas sobre algumas madeiras úteis do Sul de Minas. Revista Florestal, v.7, n. único, p.7-16, 1948.

CARVALHO, P. E. R. Espécies florestais brasileiras: potencialidades e uso da madeira. Brasília: Embrapa-CNPF/SPI, 1994. 640p.
FLEMMING, D. M.; GONÇALVES, M. B.

Cálculo A: funções, limite, derivação, integração. 5.ed. São Paulo: Makron, 1992. 617p.

FONTES, B. P. D.; DAVIDE, L. C.; DAVIDE, A. C. Fisiologia e citogenética de sementes envelhecidas de Araucaria angustifolia.

Ciência e Agrotecnologia, v.25, n.2, p.346355, 2001.

FUNDAÇÃO DE PESQUISAS FLORESTAIS DO PARANÁ - FUPEF. Conservação do bioma floresta com araucária: relatório final diagnóstico dos remanescentes florestais. Curitiba: 2001.2v.

GUBERT FILHO, F. Proposta para a criação de um sistema de unidades de conservação da Araucaria angustifolia no Estado do Paraná. In:

CONGRESSO FLORESTAL BRASILEIRO, 6., 1990, Campos do Jordão. Anais... São Paulo: Sociedade Brasileira de Silvicultura, 1990. v.3. p.287-300. (Publicado na Silvicultura, n. 42, 1990)

INSTITUTO BRASILEIRO DE GEOGRAFIA E ESTATISTICA - IBGE. Diretoria de Geociências. Departamento de Recursos Naturais e Estudos Ambientais (Rio de Janeiro, RJ). Manual técnico da vegetação brasileira. Rio de Janeiro: 1992. 92 p. (IBGE. Série Manuais Técnicos em Geociências, 1).

KRÜGEL, E. Reflorestar é preservar. Florianópolis: Souza Cruz, 1992.46p.

LORENZI, H. Árvores brasileiras: manual de identificação e cultivo de plantas nativas do Brasil. 3.ed. Nova Odessa: Plantarum, 2000. 370p.

MAtTos, J. R. O pinheiro brasileiro. 2.ed. Lages: Artes Gráficas Princesa, 1994. 225p.

MEYER, P. L. Probabilidade: aplicações à estatística. Rio de Janeiro: 1974. 391p.

PARANÁ. Secretaria de Estado do Meio Ambiente. Lista vermelha de plantas ameaçadas de extinção no Estado do Paraná. Curitiba: 1995.

REITZ, R.; KLEIN, R. M. Araucariaceae. Itajaí: Herbário Barbosa Rodrigues, 1966. 29p.

R. Árvore, Viçosa-MG, v.33, n.5, p.865-872, 2009 
REITZ, R.; KLEIN, R. M.; REIS, A. Projeto madeira do Rio Grande do Sul. Porto Alegre: SUDESUL, 1988. 528p.

SHAAF, L. B. et al.. Alteração na estrutura diamétrica de uma Floresta Ombrófila Mista no período entre 1979 e 2000. Revista Árvore, v.30, n.2, p.283-295, 2006.

SCHUMACHER, M. V. et al. Produção de serapilheira em uma floresta de Araucaria angustifolia (Bertol.) Kuntze no município de Pinhal Grande-RS. Revista Árvore, v.28, n.1, p.29-37, 2004.

SILVA, E. Q. Nova função densidade de probabilidade aplicável à Ciência Florestal. 2003. 98f. Tese (Doutorado em Engenharia Florestal) Universidade Federal do Paraná, Curitiba, 2003.
SOLÓRZANO-FILHO, J. A.; KRAUS, J. E. Breve história das matas de araucária. In: INTERNATIONAL CONGRESS AND EXHIBITION ON FORESTS, 5., 1999, Curitiba. Forest 99. Rio de Janeiro: Biosfera, 1999. p.37-40.

STEVENSON, W. J. Estatística aplicada à administração. São Paulo: Harbra, 1981.495p.

SUITER FILHO, W. Conservação de sementes de Araucaria angustifolia (Bert.) O. Ktze. Piracicaba: Escola Superior de Agricultura Luiz de Queiroz, 1966. 15p. (Não Publicado)

VIEIRA, S. Introdução à Bioestatística. 2.ed. Rio de Janeiro: Campus, 1991. 